\title{
Self-Assembly of Rod-Coil Block Copolymers for Photovoltaic Applications
}

\author{
Nicolas Sary, ${ }^{1}$ Laurent Rubatat, ${ }^{1}$ Cyril Brochon, ${ }^{2}$ Georges Hadziioannou, ${ }^{2}$ \\ Raffaele Mezzenga*1,3
}

Summary: Two different approaches to obtain electron donor-acceptor interfaces via self-assembly of block copolymer systems are discussed, where the donor domains are formed by a $\pi$-conjugated rod-like polymer and the acceptor domains result from a coiled polymer modified by $\mathrm{C}_{60}$ fullerenes. In the first strategy, $\mathrm{C}_{60}$ is chemically grafted onto the coil polymer, typically a statistical copolymer of styrene and chloromethyl styrene. This has as major effect the increase in molecular weight and volume fraction of the coil block, which can markedly perturb the self-assembled block copolymer final morphologies and eventually suppress any microseparated nanostructure in favour of fully isotropic homogeneous phases. We discuss how the presence of free homopolymer rods in the system can help recovering a microphase separated morphology suitable for photovoltaic applications. In the second approach we discuss the poly(diethylhexyl-p-phenylenevinylene-b-4-vinylpyridine) (PPV-P4VP) rod-coil block copolymer system and we argue how supramolecular interactions among P4VP and free $C_{60}$ can be exploited to blend rod-coil block copolymers and $C_{60}$ preserving the original lamellar phase.

Keywords: $\mathrm{C}_{60}$; fullerene; P4VP; poly(diethylhexyl-p-phenylenevinylene); poly-4vinylpyridine; PPV; rod-coil block copolymer

\section{Introduction}

Conjugated polymers, such as poly(diethylhexyl-p-phenylenevinylene) (PPV) or poly(thienylene vinylene) (PTV) have been successfully used to build photovoltaic devices where the active layer, a thin film of the polymer, is sandwiched between two electrodes. ${ }^{[1]}$ However, this design greatly limits the device efficiency: indeed, solar cells global yield not only depends on charge conduction but also on the efficiency

\footnotetext{
${ }^{1}$ Department of Physics and Fribourg Center for Nanomaterials, University of Fribourg, Ch. Musée 3, CH-1700, Fribourg, Switzerland E-mail: raffaele.mezzenga@unifr.ch

${ }^{2}$ Laboratoire d'Ingénierie des Polymères pour les Hautes Technologies, UMR 7165, Université Louis Pasteur, Ecole Européenne de Chimie, Polymères et Matériaux, 25, Rue Becquerel, 67087 Strasbourg, France

${ }^{3}$ Nestlé Research Center,Vers-Chez-Les-Blanc,1000 Lausanne 26(Switzerland)
}

of exciton dissociation ${ }^{[2-5]}$ which is intimately related to the amount of electron donor-electron acceptor interfaces. By taking advantage of the nanostructures typical of self-assembled block copolymer systems $^{[6]}$ an efficient design of interfaces can be envisaged and, consequently, of photon harvesting efficiency. ${ }^{[7]}$ Nevertheless, although many specific types of efficient electron donor-hole conducting polymers are available today, their acceptor-electron conductor counterparts are much more difficult to realize. As a consequence, the synthesis of a fully organic block copolymer capable of templating, alone electron donor-acceptor interfaces is still very challenging, so that prototypes of organic photovoltaic devices make often use of fullerenes as electron acceptor molecules. Furthermore, in order to preserve the self-assembly and mechanical properties of polymers, fullerene is likely to be incorporated in an insulating 
polymer matrix, which again challenges the final transport properties of the device.

In what follows we discuss two different approaches which can be used to design $\mathrm{C}_{60}$ polymeric acceptor domains: covalent bonding through chemical grafting or weak bonding produced by electron transfer complex formation. The former strategy becomes possible, for instance, by using chloromethyl styrene (CMS) monomers in a PTV-b-poly(styrene-stat-CMS) block copolymer. The fullerene molecules, which can be grafted via the CMS monomers, induce however large structure modifications and increase in coil molecular weight, whose effects need to be compensated and balanced in the rod block counterpart: a possible way to solve this problem is the addition of free homopolymer rods, as will be demonstrated with block copolymers made of polystyrene (PS) as coil and either poly(diethylhexyl-p-phenylenevinylene) (PPV) or poly(thienylene vinylene) (PTV) as rods. The second approach discussed will consist in using supramolecular redox interactions between poly(4-vinylpyridine) (P4VP), and $\mathrm{C}_{60}$, to design a PPV: $\mathrm{C}_{60}$ electron acceptor domain, when the P4VP is used as the coil block of PPV-P4VP block copolymer.

\section{Covalent Bonding}

Although PPV has shown some limitations considering its photovoltaic efficiency and stability, its relatively easy and well controlled synthesis make it a good alternative to more complex rod polymers such as polythiophenes. Here the PPV-b-PS is used as a model to study rod-coil/rod polymer blends and the influence of free homopolymer rods on the self-assembly behaviour. This study is motivated by the fact that, once a rod-coil system capable of selfassembling into lamellar phases has been designed, the grafting of $\mathrm{C}_{60}$ onto the coil block can result in the loss of the microphase separated morphology. This is demonstrated, for example, in the case of the PTV-b-P(S-co-CMS) system shown in Figure 1. The synthesis of this block copolymer, (PTV DPn =9, P(S-co-CMS) $\mathrm{Mn}=13500$, $\mathrm{PDI}=3.2)$ has been reported elsewhere. ${ }^{[8]}$ As shown by Small Angle XRay Scattering, the ungrafted block copolymer exhibits a structure peak corresponding to a pattern period of $5 \mathrm{~nm}$, which matches the double contour length of the rod used, indicating a PTV bilayer. However, this structure peak is lost when fullerene is grafted onto the $\mathrm{P}(\mathrm{S}-\mathrm{co}-$

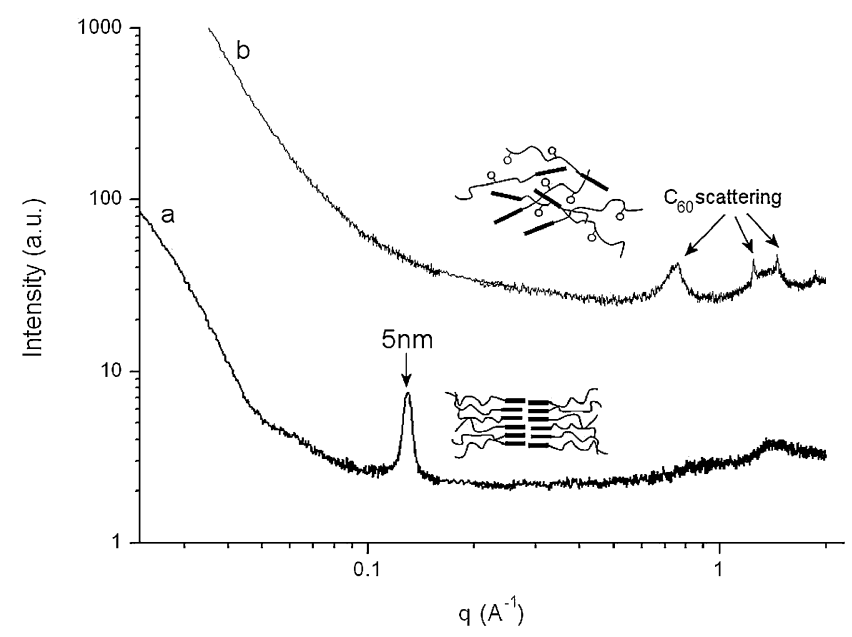

Figure 1.

SAXS diffractogram of (a) PTV-P(S-S-CMS) and (b) PTV-P(S-S-CMS) grafted with $\mathrm{C}_{60}$. When fullerene is grafted onto the coil block the $5 \mathrm{~nm}$ period corresponding to a PTV bilayer is lost. 

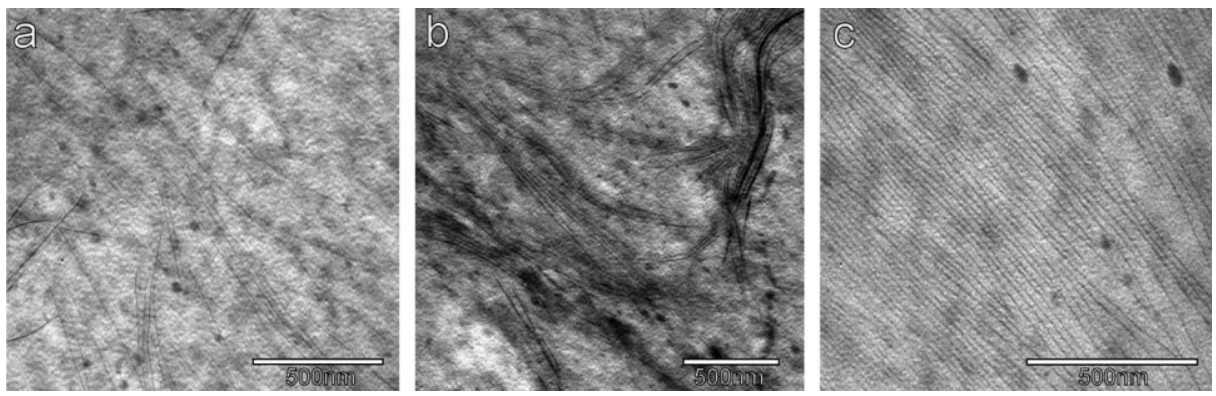

\section{Figure 2.}

TEM pictures of a PS-PPV with total $\mathrm{Mn}=8000 \mathrm{~g} / \mathrm{mol}$ and PDI $=1.52$, a) pure PS-PPV block copolymer b) PS-PPV block copolymer blended with $5 \%$ and c) $10 \%$ rod homopolymer. The addition of PPV homopolymer increases the volume of the lamellar structure at the expense of the isotropic phase.

CMS): the SAXS diffractogram only exhibit the high angle $\mathrm{C}_{60}$ characteristic peaks (Figure 1).

This observation suggests that the steric constrains induced by grafted fullerene drive the system into the isotropic phase. In order to relax the high stretching of coil chains, and thus to recover the original microphase segregated morphology, one possible option is to blend free rod homopolymers with the same block copolymer. ${ }^{[9]}$ The feasibility of this approach is demonstrated on a similar rod-coil block copolymer consisting of a PPV rod block and PS coil. In this system, the rod Mn is maintained fixed at $3400 \mathrm{~g} / \mathrm{mol} \quad(\mathrm{PDI}=1.2)$, while the total molecular weight is allowed to vary between $\mathrm{Mn}=6400 \mathrm{~g} / \mathrm{mol}(\mathrm{PDI}=$ $1.30)$ and $8700 \mathrm{~g} / \mathrm{mol}(\mathrm{PDI}=1.45) .{ }^{[9]}$ Transmission electron microscopy on pure PSPPV copolymers shows a sharp transition from lamellar to isotropic phase, without evidence of intermediate hexagonal or spherical phases. This transition occurs at about $50 \%$ coil to rod volume fraction, which considering the low rod molecular weight and the quick gain in coil molecular weight when $\mathrm{C}_{60}$ is grafted strongly restricts possible molecular architecture designs. The alternative to reduce PS coil size is the increase of PPV volume fraction: here, again, the synthetic efforts needed to afford rods blocks longer that 10 units is far from trivial. As a valuable method to solve this problem, Figure 2 shows, how starting from an isotropic phase, a lamellar phase with long-range order can be achieved by only blending free homopolymer PPV rods of identical molecular weight as the rod blocks. Furthermore, as can be seen, the order of the lamellar phase is directly dependent on the amount of PPV rod added. We argue that the same procedure can be applied to systems such as PPV-P(SCMS) or PTV-P(S-CMS) grafted with $\mathrm{C}_{60}$ to recover the lamellar structure suppressed by the grafting of fullerene.

\section{P4VP/Fullerene Complex}

The second possible approach towards the design of electron donor/acceptor interfaces consists in blending $\mathrm{C}_{60}$ into a coil host phase acting as a "chaperone" domain. The main advantage in such a strategy is the versatility of design: on the one hand the amount of active electron acceptor molecules can be easily varied without the need of new synthesis, while on the other, the optimal amount of $\mathrm{C}_{60}$ to guarantee both optoelectronic and structural properties can be easily found. In the following a PPV-P4VP block copolymer with 55\% coil volume fraction is used (Figure 3a), as polyvinylpyridines have indeed been shown to act as chaperones for both electron conductive polymers ${ }^{[10]}$ and fullerenes. ${ }^{[1]}$ 

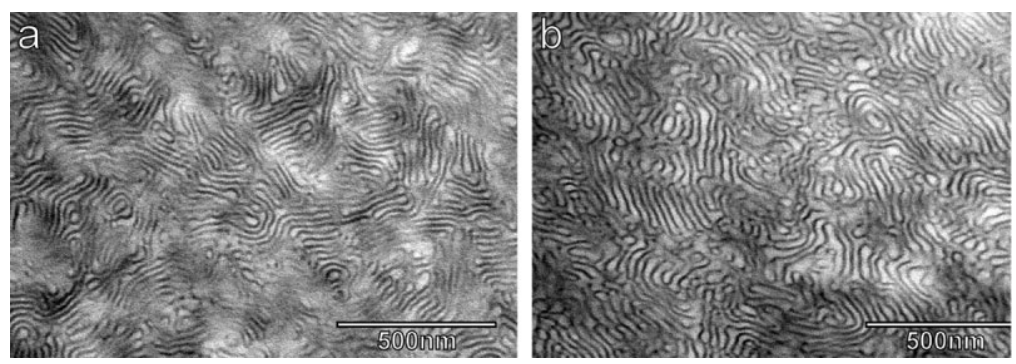

Figure 3.

TEM pictures of (a) pure PPV-P4VP copolymer and (b) with $10 \mathrm{w} \% \mathrm{C}_{60}$ complexed annealed at $180{ }^{\circ} \mathrm{C}$ for $16 \mathrm{~h}$. The P4VP phase is stained with iodine and in both cases the system exhibits clear lamellar structure with typical persistence length of the order of a few hundredths nanometres.

To form the acceptor domains via redox charge transfer reactions between P4VP and $\mathrm{C}_{60}$, the polymer is complexed in solution with $5 \%, 10 \%$ and $15 \%$ by weight of $\mathrm{C}_{60}$ (fractions are given with respect to the P4VP phase). None of these blend exhibits macroscopic phase separation or nanostructures after solvent evaporation. In order to obtain a microphase separated morphology, $16 \mathrm{~h}$ annealing at $180^{\circ} \mathrm{C}$ in high vacuum chamber were performed on the samples. In samples containing $5 \mathrm{w} \%$ and $10 \quad \mathrm{w} \% \quad \mathrm{C}_{60}$ a lamellar structure (Figure $3 b$ ) is successfully recovered. The presence of $\mathrm{C}_{60}$ however restricts the annealing temperature and thus prevents achieving those long range order lamellar structures typical of PPV-P4VP samples annealed beyond the liquid crystalline order-disorder transition temperatures of PPV rods. ${ }^{[12]}$ Due to the weak character of fullerene-P4VP bond, annealing at higher temperature causes $\mathrm{C}_{60}$ to form macroscopic phase separated domains. Annealing above polymer $\mathrm{T}_{\mathrm{g}}$ on samples bearing larger volume fractions of $\mathrm{C}_{60}$, e.g. $>15 \%$ causes a macroscopic phase separation to occur, with micron-size $\mathrm{C}_{60}$ droplets dispersed in a block copolymer continuous phase with morphology very similar to that observed for the pure block copolymer.

Although the lamellar long-range order depicted in Figure $3 b$ is lower compared to Figure $3 \mathrm{a}$, these morphologies remain suitable for designing an active polymer layer with large electron donor/acceptor interfaces in between electrodes of photovoltaic cells. Yet, the maximum amount of $\mathrm{C}_{60}$ which can be homogeneously blended to the P4VP domain using this technique is $10 \mathrm{w} \%$, corresponding to an overall volume fraction in the block copolymer of $6.5 \%$. It remains to be confirmed whether this amount can guarantee the necessary optoelectronic properties for efficient photovoltaic cells.

\section{Conclusions}

We have shown that polymer based donoracceptor interfaces can be built taking advantage of the self-assembly process typical of suitably designed rod-coil block copolymers. The acceptor properties can be tuned by either covalently bonding fullerene units to a coil polymer block, or blending it to a chaperone block through weak interactions. A procedure based on blending free homopolymer PPV rods in PPV-PS block copolymers has been reported and been proposed as a viable strategy to design interfaces in $\mathrm{PTV}-\mathrm{P}(\mathrm{S}$ CMS $)-\mathrm{C}_{60}$ and PPV-P(S-CMS)- $\mathrm{C}_{60}$ systems of use in photovoltaic devices. We have also reported on the first promising supramolecular system based on PPV-P4VP rod-coil systems blended with $\mathrm{C}_{60}$. This route enables versatile design of the $\mathrm{C}_{60}$ concentration in the P4VP domains yielding large interfaces between electron donor and electron acceptor domains, which is a 
crucial requirement for photovoltaic applications.

[1] R. A. Segalman, C. Brochon, G. Hadziioannou, S.-S. Sun, N. S. Sariciftci, Eds., Taylor \& Francis, 2005, p. 403. [2] A. J. Heeger, Reviews of Modern Physics 2001, 73, 681-700.

[3] D. R. M. Williams, G. H. Fredrickson, Macromolecules 1992, 25, 3561-3568.

[4] J. T. Chen, E. L. Thomas, C. K. Ober, S. S. Hwang, Macromolecules 1995, 28, 1688-1697.

[5] J. T. Chen, E. L. Thomas, C. K. Ober, G. P. Mao, Science 1996, 273, 343-346.

[6] L. Leibler, Macromolecules 1980, 13, 1602-1617.
[7] B. de Boer, U. Stalmach, P. F. van Hutten, C. Melzer, V. V. Krasnikov, G. Hadziioannou, Polymer 2001, 42, 9097-9109.

[8] K. Van De Wetering, C. Brochon, C. Ngov, G. Hadziioannou, Macromolecules 2006, 39, 4289-4297.

[9] N. Sary, R. Mezzenga, C. Brochon, G. Hadziioannou, J. Ruokolainen, Macromolecules 2007, 40, 3277-3286. [10] R. Mezzenga, J. Ruokolainen, G. H. Fredrickson, E. J. Kramer, D. Moses, A. J. Heeger, O. Ikkala, Science 2003, 299, 1872-1874.

[11] A. Laiho, R. H. A. Ras, S. Valkama, J. Ruokolainen, R. Osterbacka, O. Ikkala, Macromolecules 2006, 39, 7648-7653.

[12] N. Sary, L. Rubatat, C. Brochon, G. Hadziioannou, J. Ruokolainen, R. Mezzenga, Macromolecules 2007, 40, 6990-6997. 